Thromb Res. 2013 July ; 132(1): e48-e53. doi:10.1016/j.thromres.2013.04.005.

\title{
Hydrodynamic characterization of recombinant human fibrinogen species
}

\author{
Bertrand Raynala $^{\mathrm{a}}$, Barbara Cardinali ${ }^{\mathrm{b}, 1}$, Jos Grimbergen $^{\mathrm{c}}$, Aldo Profumo ${ }^{\mathrm{d}}$, Susan T. Lord ${ }^{\mathrm{b}}$, \\ Patrick Englanda , and Mattia Rocco ${ }^{\mathrm{d},{ }^{*}}$
}

anstitut Pasteur, Protéopole, Plate-Forme de Biophysique des Macromolécules et de leurs Interactions, CNRS UMR 3528, 25 Rue du Docteur Roux, 75724 Paris CEDEX 15, France; bertrand.raynal@pasteur.fr; patrick.england@pasteur.fr bepartment of Pathology and Laboratory Medicine, University of North Carolina at Chapel Hill, Chapel Hill, North Carolina 27599-7525, USA; susan@sucofa.com 'ProFibrix BV, Zernikedreef 9, 2333 CK, Leiden, The Netherlands; J.Grimbergen@ProFibrix.com dBiopolimeri e Proteomica, IRCCS AOU San MartinoIST, Istituto Nazionale per la Ricerca sul Cancro, Largo R. Benzi 10, I-16132 Genova, Italy; mattia.rocco@istge.it

\section{Abstract}

Introduction-Fibrinogen is a key component of the blood coagulation system and plays important, diverse roles in several relevant pathologies such as thrombosis, hemorrhage, and cancer. It is a large glycoprotein whose three-dimensional molecular structure is not fully known. Furthermore, circulating fibrinogen is highly heterogeneous, mainly due to proteolytic degradation and alternative mRNA processing. Recombinant production of human fibrinogen allows investigating the impact on the three-dimensional structure of specific changes in the primary structure.

\begin{abstract}
Methods-We performed analytical ultracentrifugation analyses of a full-length recombinant human fibrinogen, its counterpart purified from human plasma, and a recombinant human fibrinogen with both $\mathrm{Aa}$ chains truncated at amino acid 251, thus missing their last 359 amino acid residues.
\end{abstract}

Results-We have accurately determined the translational diffusion and sedimentation coefficients $\left(D_{t(20, w)}^{0}, s_{(20, w)}^{0}\right)$ of all three species. This was confirmed by derived molecular weights within $1 \%$ for the full length species, and $5 \%$ for the truncated species, as assessed by comparison with SDS-PAGE/Western blot analyses and primary structure data. No significant differences in the values of $D_{t(20, w)}^{0}$ and $s_{(20, w)}^{0}$ were found between the recombinant and purified full length human fibrinogens, while slightly lower and higher values, respectively, resulted for the

\footnotetext{
(c) 2013 Elsevier Ltd. All rights reserved

*Corresponding author: Mattia Rocco, Biopolimeri e Proteomica, IRCCS AOU San Martino-IST, c/o CBA, Largo R. Benzi 10, I-16132 Genova, Italy; tel: +39-010555-8310; telefax: +39-010555-8325; mattia.rocco@ istge.it..

${ }^{1}$ Present address: Sviluppo Terapie Innovative, IRCCS AOU San Martino-IST, Genova, Italy; b.cardinali@gmail.com.
}

Publisher's Disclaimer: This is a PDF file of an unedited manuscript that has been accepted for publication. As a service to our customers we are providing this early version of the manuscript. The manuscript will undergo copyediting, typesetting, and review of the resulting proof before it is published in its final citable form. Please note that during the production process errors may be discovered which could affect the content, and all legal disclaimers that apply to the journal pertain.

Conflict of interest: J. Grimbergen and S.T. Lord have a financial interest in ProFibrix BV. The other authors state that they have no conflict of interest. 
recombinant truncated human fibrinogen compared to a previously characterized purified human fibrinogen fragment $\mathrm{X}$ obtained by plasmin digestion.

Conclusions-Full-length recombinant fibrinogen is less polydisperse but hydrodynamically indistinguishable from its counterpart purified from human plasma. Recombinant Aa251truncated human fibrinogen instead behaves differently from fragment $X$, suggesting a role for the $\mathrm{B} \beta$ residues $1-52$ in inter-molecular interactions. Overall, these new hydrodynamic data will constitute a reliable benchmark against which models of fibrinogen species could be compared.

\section{Keywords}

Recombinant Fibrinogen; Blood Coagulation; Analytical Ultracentrifugation; Hydrodynamics

\section{INTRODUCTION}

Fibrinogen (FG) is a high molecular weight ( 340,000$)$, very elongated (length $\sim 45 \mathrm{~nm}$ ) plasma glycoprotein playing a central role in the blood coagulation system of vertebrates. FG is also implicated in various pathologies like thrombosis, hemorrhage, and cancer [1-3]. In plasma, FG is a heterogeneous mixture of several variants that differ mainly in primary amino acid structure. At the basic level, all variants are composed of two pairs each of three different chains ( $\mathrm{Aa}, \mathrm{B} \beta$ and $\gamma$ ) whose $\mathrm{N}$-termini reside in a central globular domain ("E" region), from which they symmetrically depart forming two triple coiled-coil connectors held in register by two "disulfide rings" [4-7]. At the end of each connector, after the second disulfide ring, the $\mathrm{B} \beta$ and $\gamma$ chains $\mathrm{C}$-terminals fold separately forming two outer globular domains ("D" regions), while the Aa chains revert their direction forming a fourth coiled helix at least up to residue Aa200 and Aa219 in the human and chicken species, respectively, as determined by X-ray crystallography [7-9]. The structure and location of the remaining $411 \mathrm{C}$-terminal residues (human numbering) of the $\mathrm{Aa}$ chains (AaC regions) is still undetermined, and has been alternatively proposed to behave as a free-swimming appendage [4, 8-9], or to form a fourth globular domain on top of the E region [10-14]. Only a small portion of the bovine species C-terminus (corresponding to residues Aa425502 in the human species) has been proved to be at least partially structured by NMR and other biophysical methods [15-18]. An additional unique feature of the AaC regions is the presence among species of a variable number (e.g., eleven in the human, none in the chicken) of 13-amino acids repeats [19-21], located between the end of the fourth coiled helix and the partially structured region. Furthermore, the AaC region is also highly susceptible to proteolytic degradation (in $25 \%$ of the plasma FG the C-terminus of the Aa chain is degraded on one side of the molecule, and in 5\% both Aa chains are degraded), which, coupled with other post-transcriptional and post-translational modifications like partial AaSer3 and other Ser/Thr residues phosphorylation [22], renders circulating FG highly polydisperse [23]. The N-terminal 16 and 14 amino acid residues of the $\mathrm{Aa}$ and $\mathrm{B} \beta$ chains, respectively, are cleaved off by thrombin during FG enzymatic activation yielding fibrin monomer $\left(a_{2} \beta_{2} \gamma_{2}\right)[1,2]$. The released peptides are known as fibrinopeptides $A$ and $\mathrm{B}$, respectively ( $\mathrm{FpA}$ and $\mathrm{FpB})$.

Hydrodynamic parameters, such as the infinite-dilution translational diffusion and sedimentation coefficients $\left(D_{t(20, w)}^{0}\right.$ and $\left.s_{\left(20, w^{\prime}\right)}^{0}\right)$, can be effectively used in multi-resolution modeling of biomacromolecules, helping to discriminate between alternative conformations (e.g., [24]; see also [25]). In a previous publication, we have reported the hydrodynamic and mass spectrometric characterization of a highly purified, nearly intact human plasma FG fraction (hpHMW-FG), of a controlled plasmin proteolysis human FG product lacking the AaC regions, termed fragment X (hpFrX-FG), and of chicken plasma FG (cpFG) [26]. We present here the results of new analytical ultracentrifugation sedimentation velocity (AUC- 
SV) experiments on a similar hpHMW-FG preparation, and on two human FG recombinant species, full-length (hrHMW-FG, from the commercial source ProFibrix), and with the Aa chain truncated after residue 251 (hra251-FG, prepared in our laboratories). We were able

to determine $s_{(20, w)}^{0}$ and $D_{t(20, w)}^{0}$ accurately, and complemented them with Aa chain polydispersity and Fp content analyses. Together these results establish benchmark data that are a reliable basis for further structural analyses of human natural and recombinant fibrinogen species.

\section{MATERIALS AND METHODS}

\section{General}

All chemicals were reagent grade from Merck (VWR International, Milano, Italy; http:// www.merck-chemicals.com/) or from Sigma-Aldrich (St. Louis, MO; http:// www.sigmaaldrich.com/united-states.html), unless otherwise stated, and double-distilled water was used in the preparation of all the solutions. DU640 and DU800 spectrophotometers (Beckman Coulter, Fullerton, CA; http://www.beckmancoulter.com) were used for all spectrophotometric operations. Quality control and characterization of the samples by polyacrylamide (PAA) gel electrophoresis in the presence of sodium dodecyl sulphate (SDS-PAGE) and Western blotting, followed by densitometric analyses, was done as previously extensively reported [26].

\section{Human plasma high molecular weight fibrinogen purification}

hpHMW-FG was purified by $\left(\mathrm{NH}_{4}\right)_{2} \mathrm{SO}_{4}$ fractional precipitation from lyophilized human fibrinogen (hFG; plasminogen depleted, type FIB1 from Enzyme Research Laboratories, South Bend, IN; http://www.enzymeresearch.com/), after reconstitution and dialysis into an appropriate buffer, as previously described in detail [26].

\section{Human recombinant high molecular weight fibrinogen preparation and purification}

hrHMW-PG was obtained from ProFibrix, Leiden, The Netherlands (http:// www.profibrix.com/), and was prepared and purified as previously described [27].

\section{Human recombinant Aa251 truncated fibrinogen preparation and purification}

Media with recombinant hra251-FG, having intact B $\beta$ and $\gamma$ chains and the Aa chain truncated after residue 251 [28], were purchased from the National Cell Culture Center (Biovest International, Minneapolis, MN, USA; http://www.biovest.com/) and purified by salt precipitation and immunoaffinity chromatography using the calcium-dependent FGspecific monoclonal antibody IF-1 (Kamiya Biomedical, Seattle, WA, USA; http:// www.kamiyabiomedical.com/) as described [29,30].

Briefly, hra 251-FG was precipitated from the medium overnight at $4{ }^{\circ} \mathrm{C}$ with $33 \%$ saturated $\left(\mathrm{NH}_{4}\right)_{2} \mathrm{SO}_{4}$. After centrifugation, the pellet was dissolved in loading buffer (Tris (tris(hydroxymethyl)aminomethane) $20 \mathrm{mM}, \mathrm{NaCl} 0.3 \mathrm{M}, \mathrm{pH}$ 7.4) with $10 \mathrm{mM} \mathrm{CaCl}_{2}$ and loaded onto the pre-equilibrated IF-1 column. After washing steps with Tris $20 \mathrm{mM}, \mathrm{NaCl} 1$ M, $\mathrm{CaCl}_{2} 10 \mathrm{mM}, \mathrm{pH} 7.4$, and $\mathrm{CH}_{3} \mathrm{COONa} 50 \mathrm{mM}, \mathrm{NaCl} 0.3 \mathrm{M}, \mathrm{CaCl}_{2} 10 \mathrm{mM}$, pH 6.0, hra251-FG was eluted with Tris $20 \mathrm{mM}, \mathrm{NaCl} 0.3 \mathrm{M}$, EDTA-Na $5 \mathrm{mM}, \mathrm{pH}$ 7.4, dialyzed once against loading buffer with $1 \mathrm{mM} \mathrm{CaCl}_{2}$ and then twice against Tris Buffered Saline (TBS; Tris $50 \mathrm{mM}, \mathrm{NaCl} 104 \mathrm{mM}$, aprotinin $10 \mathrm{KIU} / \mathrm{ml}$, pH 7.4). Several batches were purified. Each was tested by SDS-PAGE under reducing and non reducing conditions. Subsequently, Western blotting was performed using rabbit polyclonal antibodies to human FG (Dako Corp, code N A0080, Carpinteria, CA; http://www.dako.com/) and the monoclonal antibody Y18 (a gift from Dr. Nieuwenhuizen, Leiden, The Netherlands) 
specific for the N-terminus of the Aa-chain [31]. All batches were found to be $>97 \%$ clottable.

Different hra251-FG batches were pooled together, concentrated by ultrafiltration (Amicon Ultra-15, MWCO 100, Millipore, Billerica, MA; http://www.millipore.com) to $10 \mathrm{mg} / \mathrm{ml}$ (concentration above which hra251-FG tends to precipitate), added with $1 \mathrm{mM}$ AESBF (4(2-aminoethyl) benzenesulfonyl fluoride hydrochloride, Sigma-Aldrich) and stored at -80 ${ }^{\circ} \mathrm{C}$ until use.

\section{Fibrinopeptides analysis}

Fibrinopeptides release and analysis by RP-HPLC was done essentially as previously reported [32], with some modifications. In short, $140 \mu \mathrm{L}$ of reaction mixture contained FG species at $0.5 \mathrm{mg} / \mathrm{ml}$ and thrombin (Sigma-Aldrich) at 0.25 nominal NIH units $/ \mathrm{ml}$ in TBS. After $2 \mathrm{~h}$ of incubation at $25^{\circ} \mathrm{C}$, the reaction was stopped by immersion in boiling water for $1 \mathrm{~min}$, followed by centrifugation at $16,000 \mathrm{~g}$ for $15 \mathrm{~min}$. The supernatant was filtered on polyethylene sulfone $0.45 \mu \mathrm{m}$ pore-size filters (Millipore) and $40 \mu \mathrm{L}$ were injected on a 1.0 $\times 150 \mathrm{~mm}, 300 \AA ̊$ A pore size, $3.5 \mu \mathrm{m}$ particle size Symmetry $300 \mathrm{C} 18$ column, protected by a Symmetry 300 C18 $2.1 \times 10 \mathrm{~mm}$ pre-column (Waters Corp., Milford, MA, USA; http:// www.waters.com). The HPLC set-up consisted of an Agilent 1200 series system, equipped with G1379B degasser, G1376A capillary pump, G1377A micro autosampler, G1316A thermostatted column holder, and G1315B diode array detector. It was equilibrated in $97 \%$ $25 \mathrm{mM} \mathrm{CH}_{3} \mathrm{COONH}_{4}$, brought to $\mathrm{pH} 6$ with diluted $\mathrm{H}_{3} \mathrm{PO}_{4}$ (buffer A), and $3 \% 50 \mathrm{mM}$ $\mathrm{CH}_{3} \mathrm{COONH}_{4}$, brought to $\mathrm{pH} 6$ with diluted $\mathrm{H}_{3} \mathrm{PO}_{4}$ and then diluted 1:1 with $\mathrm{CH}_{3} \mathrm{CN}$ (buffer B). $\mathrm{CH}_{3} \mathrm{COONH}_{4}$ was from Fluka (Sigma-Aldrich) and $\mathrm{CH}_{3} \mathrm{CN}$ was LiChroSolv from Merck. The flow rate was $50 \mu \mathrm{l} / \mathrm{min}$, and the elution was performed in this sequence: isocratic $97 \% \mathrm{~A}-3 \% \mathrm{~B}$ for $8 \mathrm{~min}$, then three linear gradients, the first in $5 \mathrm{~min}$ to $12 \% \mathrm{~B}$, the second in $45 \mathrm{~min}$ to $27 \% \mathrm{~B}$, and the third in $6 \mathrm{~min}$ to $100 \% \mathrm{~B}$, and finally maintained at $100 \% \mathrm{~B}$ for $5 \mathrm{~min}$. The elution was monitored at $211 \mathrm{~nm}$, and the columns were thermostatted at $30^{\circ} \mathrm{C}$.

\section{Analytical Ultracentrifugation}

AUC-SV experiments were performed using a Beckman Coulter (Palo Alto, CA, USA) Optima XLI Analytical Ultracentrifuge. In each experiment, sample solutions $(400 \mu \mathrm{l})$ of various concentrations $(\sim 0.1-1.6 \mathrm{mg} / \mathrm{ml})$ and TBS buffer $(410 \mu \mathrm{l})$ were loaded into the sample and reference channels, respectively, of seven double sector, $12 \mathrm{~mm}$ optical pathlength Epon centerpiece cells. After loading, the samples were spun for a few minutes at $3000 \mathrm{rpm}$ to check for leaks and to record an absorbance scan $(200-340 \mathrm{~nm})$ used to calculate the actual sample concentration in each cell. Samples were then centrifuged at $40,000 \mathrm{rpm}$ at a constant temperature of $20.0^{\circ} \mathrm{C}$. Concentration profiles and the movement of the sedimenting boundary in the AUC cell were recorded using the Rayleigh interference optical system. AUC-SV data were analyzed by continuous size distribution analysis c(s) using Sedfit 12.0 [33] (available at http://www.analyticalultracentrifugation.com). All the $\mathrm{c}(\mathrm{s})$ distributions were calculated with a fitted frictional ratio $\mathrm{f} / \mathrm{f}_{0}$ and a maximum entropy regularization procedure with a confidence level of 0.68 . All the diffusion values were calculated from the fitted sedimentation coefficient and frictional ratio, as well as the deduced molecular mass from these parameters. Standard deviation on fitted $\mathrm{f} / \mathrm{f}_{0}$ measurement was calculated using confidence interval search in Sedphat 10.40 [33]. Theory is described in previously published work [26]. 


\section{RESULTS AND DISCUSSION}

The molecular characterization and quality control of the samples are illustrated in Figs. 13. In Fig. 1, panels A and B, SDS-PAGE analyses of non reduced and reduced samples, respectively, of hpHMW-FG, hrHMW-FG, and hra251-FG are shown. As can be seen, mainly single bands are visible in the non reduced samples, with a slight trace of higher molecular weight species only in hpHMW-FG. As for the reduced samples, hrHMW-FG has a single top band, corresponding to the Aa chain, while hpHMW-FG shows the characteristic doublet indicating a certain degree of size polydispersity. In the hra251-FG samples, the Aa chain is the last visible band migrating just above the $31 \mathrm{kD}$ marker. The $\mathrm{B} \beta$ and $\gamma$ chains appear to be indistinguishable in all samples. Since the major source of polydispersity resides in the $\mathrm{Aa}$ chain, with $\mathrm{C}$-terminal degradation products that can migrate together with the $\mathrm{B} \beta$ and $\gamma$ chains, Western blot analyses using the $\mathrm{Y}-18 \mathrm{mAb}$ specific for the N-terminal end of the Aa chain [31] was done. The results are shown in Fig. 1, panel C, where the lesser amount of C-terminally degraded Aa chains in the hrHMW-FG sample compared to hpHMW-FG samples is already evident in the two leftmost lanes (both were loaded with roughly the same total amount of protein). This is confirmed by the densitometric analyses shown in Fig. 2, where the better homogeneity of the hrHMW-FG sample (panel A) in respect to the hpHMW-FG sample (panel B) can be fully appreciated. Note that, due to the absence of the doublet in the hrHMW-FG sample, the densitometry was performed on a lane loaded with half the total amount of the other lanes (rightmost lane in Fig. 1, panel C), to avoid saturation of the main band.

The densitometry traces were then deconvolved using the EMG-GMG (exponentially modified Gaussian plus half-Gaussian modified Gaussian) chromatography function available in PeakFit v.4 (Jandel Scientific, now distributed by Systat Software Inc., Richmond, CA; http://www.sigmaplot.com/) [26], with a quadratic or a non-parametric baseline to account for the non-uniform background and avoid overestimation of the smaller peaks areas (tolerance $\sim 1 \%$ ). Typical results are shown in Fig. 2, panels C and D, but given the relatively minor effect of the smaller peaks on global computer parameters (see below), a rigorous quantization using multiple gels at different loading ratios was not attempted. For the traces examined, although many solutions could fit them almost equally well, a consensus emerged in which the main peak in hrHMW-FG (Fig. 2, panel C) derives from the contribution of a relatively small front component (blue), two main components (green and magenta, with a predominance of the slower migrating one), plus a very small trailing component (gray). The corresponding analysis for hpHMW-FG showed that the main twin peaks have a reduced amount or even lacked the front component (a small bump is observed in a compatible position), while the main components included also the small trailing component seen in hrHMW-FG, all three now in similar proportions (Fig. 2, panel D; see also Table 1). By comparison with the results of mass-spectrometry and SDS-PAGE/ Western blot analyses on similar FG samples previously carried out in our labs [26,34], we could assign the two main peaks in hrHMW-FG and hpHMW-FG to Aa 1-610 and Aa 1-601, while the very small trailing peak in hrHMW-FG, becoming the third main peak in hpHMW-FG, is likely Aa 1-583. As for the front component in hrHMW-FG, it could correspond to the 66,942 mass that we previously found in small amounts when analyzing hpHMW-FG [26], and in both cases it might represent a mixture of the Aa 1-610 Oglycosylated forms (plus phosphorylated serines in hpHMW-FG, see below) recently shown to represent $1-13 \%$ of human plasma FG [35]. Its near absence in the current hpHMW-FG preparation likely results from purification variability. The other peaks corresponding to Cterminally degraded forms of Aa chains where found to be positioned at the same locations in both samples, and were assigned, again by comparison with our previous results and for hrHMW-FG with densitometry of lanes loaded with more material (data not shown), to Aa chains species down to Aa1-424. The final results of these analyses are reported in Table 1, 
together with the molecular masses, extinction coefficients $\left(\mathrm{E}^{280}\right)$ and partial specific volumes $(\bar{v})$ calculated from the composition of the polypeptides using PROMOLP [36]. No densitometric analyses were conducted on the hra251-FG sample, as a single Aa chain band was evident. Note that the computed values for the hrHMW-FG and hpHMW-FG Aa chain species differ slightly because, as will be shown next, the hrHMW-FG apparently completely lacks the phosphorylation on the FpA which is normally present in $20 \%$ of the chains [37]. Note also that the Aa chain C-terminally degraded species are likely to be overestimated due to non-linearity effects in the Western blots staining, and for the confounding effect of baseline noise on the smaller peaks. With these considerations in mind, and assuming totally undegraded $\mathrm{B} \beta$ and $\gamma$ chains, we report the global molecular parameters computed for our three FG species in Table 2.

To further characterize our samples, we have studied the thrombin-induced fibrinopeptides release. The results are shown in Fig. 3, with the peaks identified in panel C. Very similar elution patterns were observed for hra251-FG (panel A), hrHMW-FG (panel B), and hpHMW-FG (panel C), with the two main forms present in the expected amounts. However, some relevant differences were found in both the degraded and post-translational modified forms of FpA, such as the absence of the FpAY form lacking the N-terminal alanine residue in both the recombinant proteins, and of the phosphorylated FpAP form in the hrHMW-FG sample alone. Instead, the degraded (desArgB lacking the C-terminal arginine residue, FpBY2 lacking the $\mathrm{N}$-terminal pyroglutamic acid and glycine residues) and deamidated $(\mathrm{N} \rightarrow \mathrm{D})$ forms of $\mathrm{FpB}[32]$ appears to be present in similar amounts in all species. While the absence of FpAP in hrHMW-FG could be due to the particular expression system used [27], the lack of FpAY in both recombinant species is likely due to in vivo processes of potential physiological significance [37].

The results of the sedimentation velocity analyses are globally reported in Fig. 4 as concentration-dependence plots of $1 / s_{(20, w)}\left(\right.$ panel A) and $\left(1+k_{S} c\right) \times D_{t(20, w)}($ panel B), respectively, and the derived values are reported in Table 3. As can be seen in Fig. 4, most of the samples yielded very good results, as indicated by the relatively small error bars; only the $D_{t(20, w)}$ data for hra251-FG had greater uncertainties. The $1 / s_{(20, w)}$ vs. $c$ plots of hrHMW-FG and hpHMW-FG are practically superimposable, while a slight difference is noticeable in the $\left(1+k_{S} c\right) \times D_{t(20, w)}$ vs. $c$ plots for the same two species. The extrapolated $\left\langle s_{(20, w)}^{0}\right\rangle_{w}$ and $\left\langle D_{t(20, w)}^{0}\right\rangle_{z}$ are thus very similar for hrHMW-FG and hpHMW-FG (Table 3), and in excellent agreement with literature values for unfractionated FG [5, 38-41]. As a result, the weight-average molecular weights $\langle M\rangle_{W}$ computed from the $\left\langle s_{(20, w)}^{0}\right\rangle_{W}$ and $\left\langle D_{t(20, w)}^{0}\right\rangle_{z}$ values via the Svedberg equation using the calculated ${ }_{v}$ of Table 2 , are also in excellent agreement (within 1\%) with those computed from the putative composition of the hrHMW-FG and hpHMW-FG samples. As for hra251-FG, the $\left\langle s_{(20, w)}^{0}\right\rangle_{w}$ and $\left\langle D_{t(20, w)}^{0}\right\rangle_{z}$ values are $\sim 4 \%$ higher and $\sim 12 \%$ lower, respectively, than those we previously found for hpFrX-FG [26]. Comparing the new data with literature values for fragment $\mathrm{X}$, the $\left\langle s_{(20, w)}^{0}\right\rangle_{w}$ value matches well [42], while the $\left\langle D_{t(20, w)}^{0}\right\rangle_{z}$ value is lower [43]. The slopes of the $1 / s_{(20, w)}$ vs. $c$ plots are also very similar for all the three species, leading to apparent sedimentation concentration dependence "Grálen" coefficients $k_{S}$ of the same magnitude. The positive $k_{s}$ value for hra251-FG is in contrast with the slight negative value we previously found for hpFrX-FG [26], and could reflect different thermodynamic behaviors of the two species. Furthermore, practically identical negative values for the second virial coefficient term $B M 1$ were derived from the $\left(1+k_{s} c\right) \times D_{t(20, w)}$ vs. $c$ plots for hrHMW-FG and hpHMW-FG, indicating preferential interaction between the macromolecules, while only a very slight concentration dependence was apparent for hra251-FG, again in contrast 
with what we reported for hpFrX-FG [26]. In any case, we preferred to average all the individual $D_{t(20, w)}$ values to estimate $\left\langle D_{t(20, w)}^{0}\right\rangle_{z}$ for this species. The resulting $\langle M\rangle_{W}$ for hra251-FG is $\sim 5 \%$ higher than the one calculated from its putative composition, which is still satisfactory, given the uncertainty in the $\left\langle D_{t(20, w)}^{0}\right\rangle_{z}$ value.

In conclusion, we have accurately determined the sedimentation and diffusion coefficients of a well-characterized full length, intact recombinant human fibrinogen, and of its counterpart purified from human plasma. No significant differences were found between the recombinant and the plasma derived HMW-FGs, indicating a substantial overall structural identity between the two samples. In addition, the recombinant full-length fibrinogen had a better sample homogeneity. All these findings further confirm the suitability of recombinant full-length fibrinogen species for structural studies, and they are of relevance for their potential biotechnological/biomedical applications. We also determined the hydrodynamic parameters for a recombinant fibrinogen with a truncated Aa chain (hra251-FG) and compared them with those of hpFrX-FG [26]. Although less accurately determined, the observed differences in the $\left\langle D_{t(20, w)}^{0}\right\rangle_{z}$ and $k_{S}$ values could stem from differences at the molecular level, like the lack of both B $\beta$ 1-52 segments in hpFrX-FG. This suggests a role for these regions in FG-FG inter-molecular interactions. In any case, given the difficulties in obtaining complete atomic-level structures of fibrinogen, these new hydrodynamic data will constitute a reliable benchmark against which multi-resolution models could be compared.

\section{Acknowledgments}

We thank J. Koopman (ProFibrix, The Netherlands) for reviewing the manuscript. Work partially supported by a grant from MIUR, Italy (PRIN 2008HAFF7X), and by NIH grant HL031048 (to S.T.L.).

\section{Abbreviations}

$\begin{array}{ll}\text { FG } & \text { fibrinogen } \\ \text { FpA } & \text { fibrinopeptide A } \\ \text { FpB } & \text { fibrinopeptide B } \\ \text { AUC-SV } & \text { analytical ultracentrifugation sedimentation velocity } \\ \text { hpHMW-FG } & \text { human plasma high molecular weight FG } \\ \text { hpFrX-FG } & \text { human plasma FG fragment X } \\ \text { cpFG } & \text { chicken plasma FG } \\ \text { hrHMW-FG } & \text { human recombinant high molecular weight FG } \\ \text { hra251-FG } & \text { human recombinant FG with Aa chains truncated after residue 251 }\end{array}$

\section{REFERENCES}

[1]. Doolittle, RF. The molecular biology of fibrin. In: Stamatoyannopoulos, G.; Majerus, PW.; Perlmutter, RM.; Varmus, H., editors. The Molecular Basis of Blood Diseases. third ed.. Saunders; Philadelphia: 2000. p. 719-39.

[2]. Blombäck B. Fibrinogen and fibrin--proteins with complex roles in hemostasis and thrombosis. Thromb Res. 1996; 83:1-75. [PubMed: 8837305]

[3]. Boccaccio C, Medico E. Cancer and blood coagulation. Cell Mol Life Sci. 2006; 63:1024-27. [PubMed: 16612563]

[4]. Doolittle RF. Fibrinogen and fibrin. Annu Rev Biochem. 1984; 53:195-229. [PubMed: 6383194] 
[5]. Weisel JW. Fibrinogen and fibrin. Adv Protein Chem. 2005; 70:247-99. [PubMed: 15837518]

[6]. Doolittle RF, Goldbaum DM, Doolittle LR. Designation of sequences involved in the "coiled-coil" interdomainal connections in fibrinogen: constructions of an atomic scale model. J Mol Biol. 1978; 120:311-25. [PubMed: 642011]

[7]. Spraggon G, Everse SJ, Doolittle RF. Crystal structures of fragment D from human fibrinogen and its crosslinked counterpart from fibrin. Nature. 1997; 389:455-62. [PubMed: 9333233]

[8]. Yang Z, Kollman JM, Pandi L, Doolittle RF. Crystal structure of native chicken fibrinogen at 2.7 Å resolution. Biochemistry. 2001; 40:12515-23. [PubMed: 11601975]

[9]. Kollman JM, Pandi L, Sawaya MR, Riley M, Doolittle RF. Crystal structure of human fibrinogen. Biochemistry. 2009; 48:3877-86. [PubMed: 19296670]

[10]. Mosesson MW, Hainfeld J, Wall J, Haschemeyer RH. Identification and mass analysis of human fibrinogen molecules and their domains by scanning transmission electron microscopy. J Mol Biol. 1981; 153:695-718. [PubMed: 7338923]

[11]. Medved' LV, Gorkun OV, Privalov PL. Structural organization of C-terminal parts of fibrinogen Aa-chains. FEBS Lett. 1983; 160:291-95. [PubMed: 6224704]

[12]. Erickson, HP.; Fowler, WE. Electron microscopy of fibrinogen, its plasmic fragments and small polymers. In: Mosesson, MW.; Doolittle, RF., editors. Molecular Biology of Fibrinogen and Fibrin. Vol. vol. 408. Annals of the New York Academy of Sciences; New York: 1983. p. 146-63.New York Academy of Sciences

[13]. Weisel JW, Stauffacher CV, Bullit TE, Cohen C. A model for fibrinogen: domains and sequence. Science. 1985; 230:1388-91. [PubMed: 4071058]

[14]. Litvinov RI, Yakovlev S, Tsurupa G, Gorkun OV, Medved L, Weisel JW. Direct evidence for specific interactions of the fibrinogen aC-domains with the central $\mathrm{E}$ region and with each other. Biochemistry. 2007; 46:9133-42. [PubMed: 17630702]

[15]. Tsurupa G, Tsonev L, Medved L. Structural organization of the fibrin(ogen) aC-domain. Biochemistry. 2002; 41:6449-59. [PubMed: 12009908]

[16]. Burton RA, Tsurupa G, Medved L, Tjandra N. Identification of an ordered compact structure within the recombinant bovine fibrinogen aC-domain fragment by NMR. Biochemistry. 2006; 45:2257-66. [PubMed: 16475814]

[17]. Burton RA, Tsurupa G, Hantgan RR, Tjandra N, Medved L. NMR solution structure, stability, and interaction of the recombinant bovine fibrinogen aC-domain fragment. Biochemistry. 2007; 46:8550-60. [PubMed: 17590019]

[18]. Tsurupa G, Hantgan RR, Burton RA, Pechik I, Tjandra N, Medved L. Structure, stability, and interaction of the fibrin(ogen) aC-domains. Biochemistry. 2009; 48:12191-201. [PubMed: 19928926]

[19]. Doolittle RF, Watt KWK, Cottrell BA, Strong DD, Riley M. The amino acid sequence of the achain of human fibrinogen. Nature. 1979; 280:464-8. [PubMed: 460425]

[20]. Henschen, A.; Lottspeich, F.; Kehl, M.; Southan, C. Covalent structure of fibrinogen. In: Mosesson, MW.; Doolittle, RF., editors. Molecular Biology of Fibrinogen and Fibrin. Vol. vol. 408. Annals of the New York Academy of Sciences; New York: 1983. p. 28-43.New York Academy of Sciences

[21]. Weissbach L, Grieninger G. Bipartite mRNA for chicken a-fibrinogen potentially encodes an amino acid sequence homologous to $\beta$ - and $\gamma$-fibrinogens. Proc Natl Acad Sci USA. 1990; 87:5198-202. [PubMed: 2367530]

[22]. Zahedi RP, Lewandrowski U, Wiesner J, Wortelkamp S, Moebius J, Schütz C, Walter U, Gambaryan S, Sickmann A. Phosphoproteome of resting human platelets. J Proteome Res. 2008; 7:526-34. [PubMed: 18088087]

[23]. Mosesson, MW. Fibrinogen heterogeneity. In: Mosesson, MW.; Doolittle, RF., editors. Molecular Biology of Fibrinogen and Fibrin. Vol. vol. 408. Annals of the New York Academy of Sciences; New York: 1983. p. 97-113.New York Academy of Sciences

[24]. Rosano C, Rocco M. Solution properties of full-length integrin $a_{I I b} \beta_{3}$ refined models suggest environment-dependent induction of alternative bentextended resting states. FEBS J. 2010; 277:3190-202. [PubMed: 20584077] 
[25]. Byron O. Hydrodynamic modeling: the solution conformation of macromolecules and their complexes. Methods Cell Biol. 2008; 84:327-73. [PubMed: 17964937]

[26]. Cardinali B, Profumo A, Aprile A, Byron O, Morris G, Harding SE, Stafford WF, Rocco M. Hydrodynamic and mass spectrometry analysis of nearly-intact human fibrinogen, chicken fibrinogen, and of a substantially monodisperse human fibrinogen fragment X. Arch Biochem Biophys. 2010; 493:157-68. [PubMed: 19853574]

[27]. Radulovic V, Baghaei F, Blixter IF, Samuelsson S, Jeppsson A. Comparable effect of recombinant and plasma-derived human fibrinogen concentrate on ex vivo clot formation after cardiac surgery. J Thromb Haemost. 2012; 10:1696-98. [PubMed: 22712790]

[28]. Gorkun OV, Henschen-Edman AH, Ping LF, Lord ST. Analysis of Aa251 fibrinogen: the aC domain has a role in polymerization, albeit more subtle than anticipated from the analogous proteolytic fragment X. Biochemistry. 1998; 37:5434-41.

[29]. Okumura N, Gorkun OV, Lord ST. Severely impaired polymerization of recombinant fibrinogen $\gamma$-364 Asp $\rightarrow$ His, the substitution discovered in a heterozygous individual. J Biol Chem. 1997; 272:29596-601. [PubMed: 9368024]

[30]. Gorkun OV, Veklich YI, Weisel JW, Lord ST. The conversion of fibrinogen to fibrin: recombinant fibrinogen typifies plasma fibrinogen. Blood. 1997; 89:4407-14. [PubMed: 9192765]

[31]. Koppert PW, Huijsmans CMG, Nieuwenhuizen W. A monoclonal antibody, specific for human fibrinogen, fibrinopeptide A-containing fragments and not reacting with free fibrinopeptide A. Blood. 1985; 66:503-07. [PubMed: 4040783]

[32]. Cardinali B, Damonte G, Melone L, Salis A, Tosetti F, Rocco M, Profumo A. Identification of a new truncated form and deamidation products of fibrinopeptide $\mathrm{B}$ released by thrombin from human fibrinogen. Thromb Haemost. 2006; 96:302-08. [PubMed: 16953271]

[33]. Brown PH, Schuck P. Macromolecular size-and-shape distributions by sedimentation velocity analytical ultracentrifugation. Biophys J. 2006; 90:4651-61. [PubMed: 16565040]

[34]. Profumo A, Turci M, Damonte G, Ferri F, Magatti D, Cardinali B, Cuniberti C, Rocco M. Kinetics of fibrinopeptide release by thrombin as a function of $\mathrm{CaCl}_{2}$ concentration: different susceptibility of FPA and FPB and evidence for a fibrinogen isoform-specific effect at physiological $\mathrm{Ca}^{2+}$ concentration. Biochemistry. 2003; 42:12335-48. [PubMed: 14567695]

[35]. Zauner G, Hoffmann M, Rapp E, Koeleman CA, Dragan I, Deelder AM, Wuhrer M, Hensbergen PJ. Glycoproteomic analysis of human fibrinogen reveals novel regions of O-glycosylation. J Proteome Res. 2012; 11:5804-14. [PubMed: 23050552]

[36]. Spotorno B, Piccinini L, Tassara G, Ruggiero C, Nardini M, Molina F, Rocco M. BEAMS (BEAds Modelling System): a set of computer programs for the generation, the visualization and the computation of the hydrodynamic and conformational properties of bead models of proteins. Eur Biophys J. 1997; 25:373-84. Erratum 1997;26:417.

[37]. Southan C, Thompson E, Lane DA. Direct analysis of plasma fibrinogen-derived fibrinopeptides by high performance liquid chromatography: investigation of Aa-chain N-terminal heterogeneity. Thromb Haemost. 1986; 56:193-97. [PubMed: 3643663]

[38]. Doolittle RF. Structural aspects of the fibrinogen to fibrin conversion. Adv Prot Chem. 1973; 27:1-109.

[39]. Scheraga HA, Laskowski M Jr. The fibrinogen-fibrin conversion. Adv Prot Chem. 1957; 12:1131.

[40]. Palmer GR, Fritz OG, Hallett FR. Quasielastic light-scattering studies on human fibrinogen and fibrin. I. Fibrinogen. Biopolymers. 1979; 18:1647-58.

[41]. Mosesson MW, Alkjaersing N, Sweet B, Sherry S. Human fibrinogen of relatively high solubility. Comparative biophysical, biochemical, and biological studies with fibrinogen of lower solubility. Biochemistry. 1967; 6:3279-87. [PubMed: 6056988]

[42]. Marder VJ, Shulman NR, Carroll WR. High molecular weight derivatives of human fibrinogen produced by plasmin. I. Physicochemical and immunological characterization. J Biol Chem. 1969; 244:2111-9. [PubMed: 4238527] 
[43]. Dietler, G.; Wiltzius, P.; Grolimund, P.; Kanzig, W.; Haberli, A.; Straub, PW. Fibrinogen Structure, Functional Aspects, Metabolism. Vol. vol. 2. Walter de Gruyter \& Co.; Berlin-New York: 1983. Early plasmin digestion of fibrinogen: light scattering data; p. 19-23. 


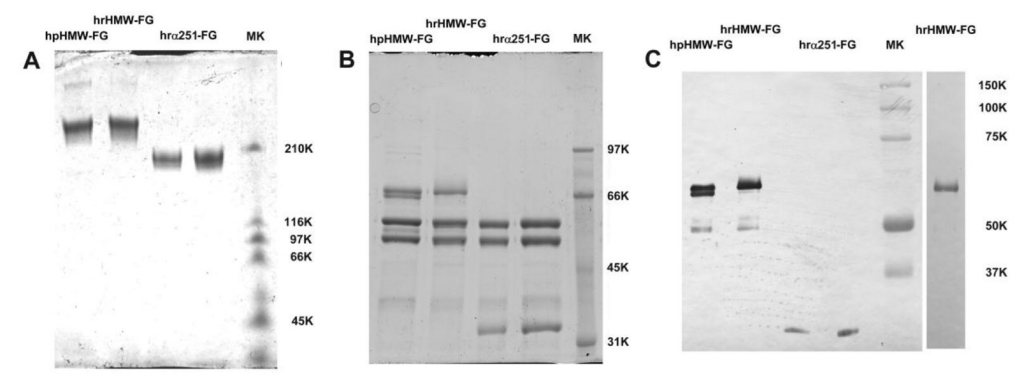

Figure 1.

SDS-PAGE and Western blot analyses of the hra251-FG, hrHMW-FG, and hpHMW-FG samples. Panel A, non-reduced samples $(3.75 \mu \mathrm{g} / \mathrm{lane})$ run on a 3.2\% PAA/8.5 M urea gel, stained with Coomassie blue (due to the non-reducing conditions of the samples, the molecular weight markers are just indicative). Panel B, reduced samples (7.5 $\mu \mathrm{g} / \mathrm{lane})$ run on a $7.5 \%$ PAA gel, stained with Coomassie blue. Panel C, Western blot of the samples in panel B $(0.5 \mu \mathrm{g} / \mathrm{lane})$ stained with the Y-18 mAb (pre-stained molecular weight markers on the rightmost lane); the additional lane on the right side was loaded with $0.25 \mu \mathrm{g}$ of hrHMW-FG. 


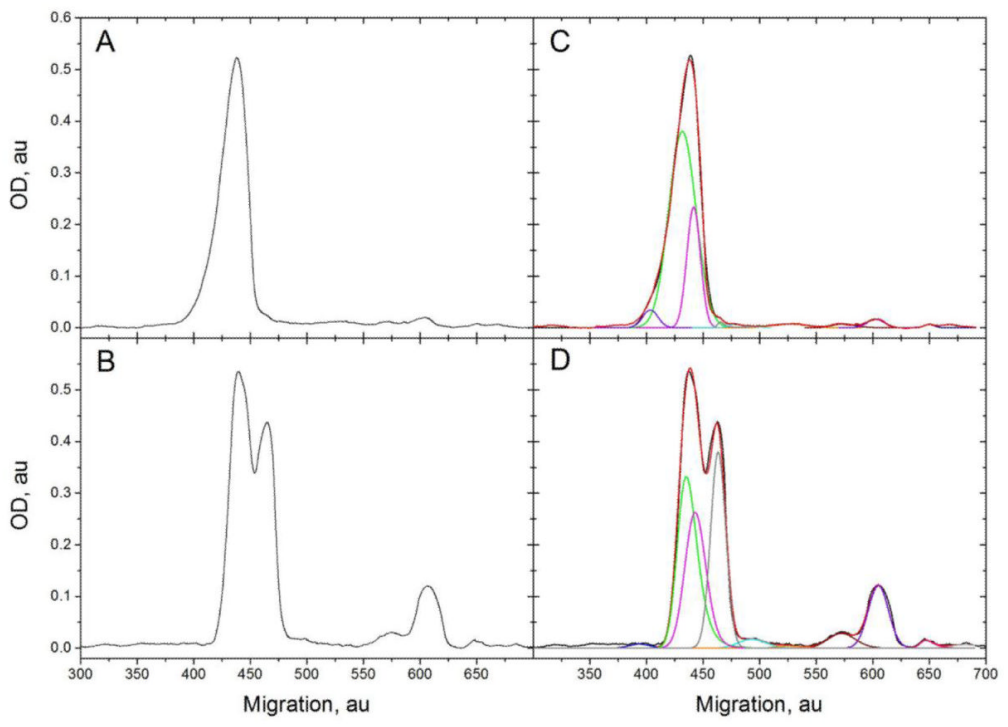

Figure 2.

Densitometry traces of the mAb Y18-stained Western blots of hrHMW-FG (panel A; rightmost lane of Fig. 1, panel C) and hpHMW-FG (panel B; leftmost lane of Fig. 1, panel C). In both panels the $y$-axis data were vertically shifted by subtracting a baseline value (no rescaling was applied). The $x$-axis values of the panel B data where horizontally shifted to find the best alignment between the main peaks in both panels. The corresponding deconvolution results are shown in panels $\mathrm{C}$ and $\mathrm{D}$, respectively. The color coding relates the resulting EMG-GMG peaks to the data in Table 1: black, original curve; red, reconstructed curve; blue, peak 1; green, peak 2; magenta, peak 3; grey, peak 4; cyan, peak 5 ; orange, peak 6; wine, peak 7; violet, peak 8; pink, peak 9. 


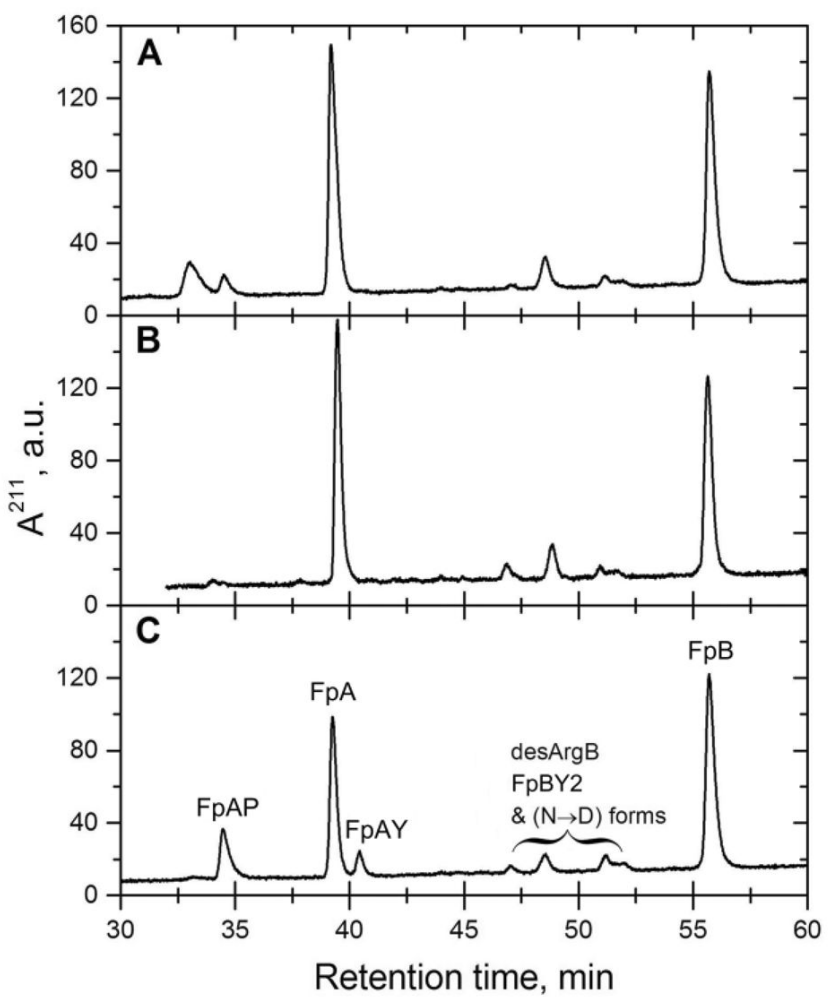

Figure 3.

RP-HPLC analysis of fibrinopeptide content of hra251-FG (panel A), hrHMW-FG (panel $\mathrm{B}$ ), and hpHMW-FG (panel C). The various Fps peaks are identified in panel C (FpAP, phosphorylated FpA; FpAY, FpA lacking the N-terminal Ala residue; desArgB, FpB lacking the $\mathrm{C}$-terminal Arg residue; FpBY2, FpB lacking the N-terminal pyroGlu and Gly residues; $\mathrm{N} \rightarrow \mathrm{D}$, deamidated forms of all FpBs; the first "peak" in panel A is a spurious signal not corresponding to any Fp species). 


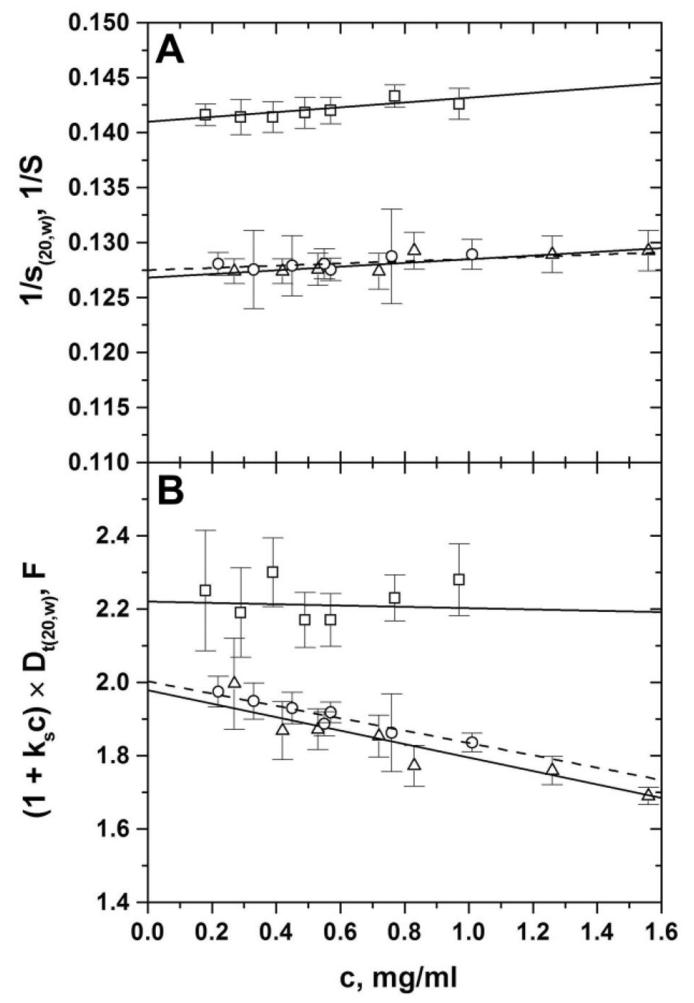

Figure 4.

Concentration-dependence plots of the reciprocal of the sedimentation coefficients (panel A) and of the diffusion coefficients (panel B) derived from the SedFit analysis of sedimentation velocity runs for hra251-FG (open squares), hpHMW-FG (open circles), and hrHMW-FG (open triangles). The straight lines are their respective weighted linear regressions (dashed, hpHMW-FG). 
Table 1

Identification of the Aa-chain species in hrHMW-FG and hpHMW-FG, their calculated molecular parameters, and relative $\%$ abundances derived from the densitometric analysis of Western blots.

\begin{tabular}{|c|c|c|c|c|c|}
\hline Peak \# & Tentative identification & Mass from composition & $\mathrm{E}^{280}\left(\mathrm{ml} \mathrm{mg} \mathrm{mg}^{-1} \mathrm{~cm}^{-1}\right)$ & $\bar{v}\left(m l g^{-1}\right)$ & Relative $\%$ (from gels) ${ }^{a}$ \\
\hline \multicolumn{6}{|c|}{ hrHMW-FG } \\
\hline 1 & Aa 1-610 (O-Gly) & 66,751 & 1.03 & 0.710 & 3.5 \\
\hline 2 & Aa 1-610 & 66,094 & 1.04 & 0.711 & 68.7 \\
\hline 3 & Aa 1-601 & 65,105 & 1.06 & 0.711 & 21.1 \\
\hline 4 & Aa $1-583$ & 63,250 & 1.09 & 0.712 & 0.8 \\
\hline 5 & Aa 1-554 & 60,022 & 1.07 & 0.713 & 0.7 \\
\hline 6 & Aa 1-528 & 57,281 & 1.12 & 0.713 & 1.7 \\
\hline 7 & Aa $1-508$ & 55,033 & 1.17 & 0.713 & 1.2 \\
\hline 8 & $\mathrm{Aa}-461$ & 50,111 & 1.28 & 0.713 & 1.9 \\
\hline 9 & Aa 1-424 & 46,223 & 1.39 & 0.712 & 0.5 \\
\hline \multicolumn{6}{|c|}{ hpHMW-FG } \\
\hline 1 & Aa 1-610 (O-Gly) & 66,782 & 1.03 & 0.710 & 0.7 \\
\hline 2 & Aa1-610 & 66,125 & 1.04 & 0.711 & 28.8 \\
\hline 3 & Aa1-601 & 65,137 & 1.06 & 0.711 & 29.3 \\
\hline 4 & Aa $1-583$ & 63,282 & 1.09 & 0.712 & 23.8 \\
\hline 5 & Aa 1-554 & 60,054 & 1.07 & 0.713 & 1.2 \\
\hline 6 & Aa $1-528$ & 57,313 & 1.12 & 0.713 & 0.5 \\
\hline 7 & Aa $1-508$ & 55,065 & 1.17 & 0.713 & 3.5 \\
\hline 8 & Aa1-461 & 50,143 & 1.28 & 0.713 & 11.2 \\
\hline 9 & Aa 1-424 & 46,255 & 1.39 & 0.712 & 1.0 \\
\hline
\end{tabular}

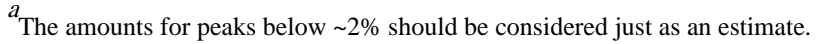


Table 2

Calculated molecular properties of the hra251-FG, hrHMW-FG, and hpHMW-FG species.

\begin{tabular}{c|c|c|c}
\hline Species & Molecular weight $\left(\mathbf{g ~ m o l}^{\mathbf{- 1}}\right)$ & $\bar{v}\left(m l g^{-1}\right)$ & $\mathbf{E}^{\mathbf{2 8 0}\left(\mathbf{m l ~ m g}^{\mathbf{- 1}} \mathbf{c m}^{\mathbf{- 1}}\right)}$ \\
\hline hra251-FG & $264,410^{a}$ & $0.720^{a}$ & $1.60^{a}$ \\
hrHMW-FG & 336,694 & 0.715 & 1.53 \\
hpHMW-FG & 331,683 & 0.715 & 1.55 \\
\hline
\end{tabular}

${ }^{a}$ Values derived from the theoretical composition only. 


\section{Table 3}

Parameters derived from the analysis of the sedimentation velocity data.

\begin{tabular}{|c|c|c|c|c|c|c|c|}
\hline Sample & $<S_{(20, w)}^{0}>_{w}(\mathrm{~S})$ & $k_{s}^{\prime}\left(m l g^{-1}\right)$ & $r^{2}$ & $<D_{t(20, w)}^{0}>_{z}(\mathrm{~F})$ & BM1 $\left(\mathrm{ml} \mathrm{g}^{-1}\right)$ & $r^{2}$ & $\underset{\left.{ }^{\langle} \mathrm{mol}^{-1}\right)}{\langle M\rangle(\% \text { diff })^{a}(\mathrm{~g}}$ \\
\hline hra 251-FG & $7.09 \pm 0.02$ & $15.6 \pm 4.9$ & 0.6685 & $2.21 \pm 0.05^{b}$ & $\sim 0$ & NA & $277,976 \pm 6,287(+5.1 \%)$ \\
\hline hrHMW-FG & $7.89 \pm 0.02$ & $13.3 \pm 3.7$ & 0.7228 & $1.98 \pm 0.02$ & $-92.7 \pm-8.1$ & 0.9705 & $339,164 \pm 3,737(+0.7 \%)$ \\
\hline hpHMW-FG & $7.84 \pm 0.02$ & $7.9 \pm 5.1$ & 0.3206 & $2.00 \pm 0.01$ & $-84.1 \pm-10.0$ & 0.9342 & $333,346 \pm 1,032(+0.5 \%)$ \\
\hline
\end{tabular}

${ }^{a} \%$ difference from the sequence-based MW adjusted according to SDS-PAGE/Western blot-derived values (only for hrHMW-FG and hpHMWFG, see Tables 1 and 2);

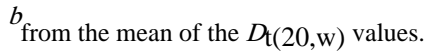

